\title{
An International Survey on the Use of Thromboprophylaxis in Urological Surgery
}

\author{
Philippe D. Violette ${ }^{a, b}$, Robin W.M. Vernooij ${ }^{c, d}$, Yoshitaka Aoki ${ }^{e}$, Arnav Agarwal $^{f}$, \\ Rufus Cartwright ${ }^{g, h}$, Yoichi Arai ${ }^{i}$, Thomas Tailly ${ }^{j}$, Giacomo Novara ${ }^{k}$, Tejan Baldeh ${ }^{a}$, \\ Samantha Craigie ${ }^{a}$, Rodney H. Breau ${ }^{l}$, Gordon H. Guyatt ${ }^{a}$, Kari A.O. Tikkinen ${ }^{m, *}$
}

${ }^{a}$ Department of Health Research Methods, Evidence and Impact, McMaster University, Hamilton, ON, Canada; ${ }^{b}$ Department of Surgery, Division of Urology, McMaster University, Hamilton, ON, Canada; ' Julius Center for Health Sciences and Primary Care, University Medical Center Utrecht, Utrecht University, Utrecht, The Netherlands; ${ }^{\mathrm{d}}$ Department of Nephrology \& Hypertension, University Medical Center Utrecht, Utrecht, The Netherlands; ${ }^{\mathrm{e}}$ Department of Urology, University of Fukui Faculty of Medical Sciences, Fukui, Japan; ${ }^{\mathrm{f}}$ Faculty of Medicine, University of Toronto, Toronto, ON, Canada; ${ }^{\mathrm{g}}$ Department of Epidemiology and Biostatistics, Imperial College London, London, UK; ${ }^{\mathrm{h}}$ Department of Urogynecology, Oxford University Hospitals NHS Trust, Oxford, UK; ${ }^{i}$ Department of Urology, Tohoku University Graduate School of Medicine, Sendai, Japan; ${ }^{\mathrm{j}}$ Division of Urology, Department of Surgery, University Hospital Ghent, Ghent, Belgium; ${ }^{k}$ Department of Surgical, Oncological, and Gastroenterological Sciences, Urology Clinic, University of Padua, Padua, Italy; ${ }^{1}$ Division of Urology, The Ottawa Hospital Research Institute and University of Ottawa, Ottawa, ON, Canada; ${ }^{\mathrm{m}}$ Departments of Urology and Public Health, University of Helsinki and Helsinki University Hospital, Helsinki, Finland

\section{Article info}

Article history:

Accepted May 27, 2020

\section{Associate Editor: Malte Rieken}

\section{Keywords:}

Bleeding

Deep vein thrombosis

Guideline

Hemorrhage

Practice variation

Prevention

Pulmonary embolism

Surgery

Thromboprophylaxis

Thrombosis

Urology

Venous thromboembolism

\begin{abstract}
Background: The use of perioperative thromboprophylaxis in urological surgery is common but not standardized.

Objective: To characterize international practice variation in thromboprophylaxis use in urological surgery.

Design, setting, and participants: We conducted a scenario-based survey addressing the use of mechanical and pharmacological thromboprophylaxis in urological cancer procedures (radical cystectomy [RC], radical prostatectomy [RP], and radical nephrectomy [RN]) among practicing urologists in Canada, Finland, and Japan. The survey presented patient profiles reflecting a spectrum of risk for venous thromboembolism; the respondents described their clinical practice.

Outcome measurements and statistical analysis: The proportion of respondents who routinely used (1) mechanical, (2) pharmacological, and (3) extended pharmacological prophylaxis was stratified by procedure. A logistic regression identified characteristics associated with thromboprophylaxis use.

Results and limitations: Of 1051 urologists contacted, 570 (54\%) participated in the survey. Japanese urologists were less likely to prescribe pharmacological prophylaxis than Canadian or Finnish urologists ( $p<0.001$ for all procedures). Canadian and Finnish urologists exhibited large variation for extended pharmacological prophylaxis for RP and RN. Finnish urologists were most likely to prescribe extended prophylaxis versus Canadian and Japanese urologists (RC 98\%, 84\%, and 26\%; Open RP 25\%, 8\%, and 3\%; robotic RP $11 \%, 9 \%$, and $0 \%$; and RN 43\%, 7\%, and 1\%, respectively; $p<0.001$ for each procedure). Less variation was found regarding the prescription of mechanical prophylaxis, which was most commonly used until ambulation or discharge. The length of hospital stay was longer in Japan and may bias estimates of extended prophylaxis in Japan.

* Corresponding author. Department of Urology, Helsinki University Hospital, Haartmaninkatu 4, Helsinki 00029, Finland. Tel. +358-50-5250971.

E-mail address: kari.tikkinen@gmail.com (Kari A.O. Tikkinen).
\end{abstract}

https://doi.org/10.1016/j.euf.2020.05.015

2405-4569/@ 2020 European Association of Urology. Published by Elsevier B.V. This is an open access article under the CC BY-NC-ND license (http:// creativecommons.org/licenses/by-nc-nd/4.0/). 
Conclusions: We found large variation in clinical practice regarding pharmacological thromboprophylaxis within and between countries. Knowledge translation of evidence-based guidelines may reduce problematic international variation in practice. Patient summary: Use of medications to decrease blood clots after urological cancer surgery differs within and between countries. Closer adherence to urology guidelines addressing the prevention of blood clots may decrease this variation and improve patient outcomes.

(C) 2020 European Association of Urology. Published by Elsevier B.V. This is an open access article under the CC BY-NC-ND license (http://creativecommons.org/licenses/

by-nc-nd/4.0/).

\section{Introduction}

Venous thromboembolism (VTE), including deep vein thrombosis and pulmonary embolism, represents a serious and sometimes fatal complication of surgery [1]. Pharmacological prophylaxis decreases the relative risk of VTE in surgical patients by approximately $50 \%$, but with an increase in the relative risk of postoperative major bleeding of 50\% [2]. Therefore, the decision to use pharmacological prophylaxis presents a tradeoff between a reduction in VTE and an increase in bleeding [3].

An additional challenge regarding the use of thromboprophylaxis in urology is international clinical practice variation [4-6]. A lack of knowledge of evidence regarding the procedure- and patient-specific baseline risks of thrombosis and bleeding, critical in making an informed decision on the use of thromboprophylaxis, may, at least in part, explain this variation $[1,2,7]$. Conflicting recommendations from different guidelines and previous lack of guidelines specific to the different urological surgeries may also contribute $[3,8]$. To further understand international variation in thromboprophylaxis use in urological surgery, we conducted an international survey (International Survey on Use of Thromboprophylaxis in Urological Surgery [ISTHMUS]). Our goal was to characterize the within- and betweencountry variation in thromboprophylaxis use for common urological procedures.

\section{Patients and methods}

\subsection{Survey}

We designed a questionnaire that consisted of three scenarios intended to elicit practice in prescribing both pharmacological and mechanical thromboprophylaxis for radical cystectomy (RC), open and robotic radical prostatectomy (RP), and radical nephrectomy (RN; Table 1). Using the patient risk factor model used in the European Association of Urology (EAU) guideline on thromboprophylaxis in urological surgery [3], the survey presented brief patient profiles that reflected a spectrum of VTE risks (Table 1). Table 2 presents the degree of VTE risk, based on systematic reviews [2], associated with each scenario. This information was not included in the survey and thus was unavailable to participants. Participants indicated their usual practice for each patient profile using response options consisting of single-answer multiple choices (Table 1). Respondents also provided their age, gender, and urologist profile (resident/consultant). A group of clinicians and methodologists generated the items in the questionnaire, which was then pilot tested and reviewed with a group of 20 board-certified urological surgeons from Canada, Finland, and Japan who assessed its face validity.
We performed this survey before the first procedure- and patientspecific thromboprophylaxis guideline in urology-the EAU guidelinewas published [3]. We invited actively practicing urologists from Canada, Finland, and Japan to complete the survey. In Canada, we invited Canadian urologists attending the annual meeting of the Canadian Urological Association (CUA), held in Ottawa in June 2015. In Finland and Japan, we identified urologists from the registers of the Finnish Urological Association and the Japanese Urological Association. In Canada, urologists completed the survey at the conclusion of the plenary sessions and at break periods throughout the conference. We collected surveys immediately upon completion and used the number of urologists attending the meeting as the denominator for calculating the response rate (the use of the number of urologists attending the meeting as the denominator likely underestimates the response rate). All urologists in Finland received an invitation to participate by a postal letter. In Japan, a sample of 500 urologists (of whom 487 proved to be eligible) were randomly identified from the national membership directory and invited, by mail, to participate. We mailed the questionnaires between August and September 2015 (first round), and mailed two rounds of reminders in October (second round) and December 2015 (third round). Furthermore,

Table 1 - Scenarios tested in the survey with response options.

Scenario 1 (cystectomy): A 65-yr-old woman who has a BMI of 36 and a personal history of VTE but is otherwise healthy is undergoing an open radical cystectomy. If you were prescribing thromboprophylaxis for this patient, what regimen would you choose most commonly?

Scenario 2 (prostatectomy): A 58-yr-old man with a BMI of 23 and no personal or family history of VTE is undergoing a radical prostatectomy without lymphadenectomy (respondents were asked to select an open or a robotic approach). If you were prescribing thromboprophylaxis for this patient, what regimen would you choose most commonly?

Scenario 3 (nephrectomy): An 80-yr-old man who has a BMI of 24 and no personal or family history of VTE is undergoing an open radical nephrectomy. If you were prescribing thromboprophylaxis for this patient, what regimen would you choose most commonly?

Response options:

Pharmacological prophylaxis:

1. No pharmacological thromboprophylaxis

2. Pharmacological thromboprophylaxis until the patient is ambulating

3. Pharmacological thromboprophylaxis for the duration of hospital stay

4. Pharmacological thromboprophylaxis for the duration of hospital stay and $\leq 2$ wk after discharge

5. Pharmacological thromboprophylaxis for the duration of hospital stay and $>2$ wk after discharge

Mechanical prophylaxis:

1. No mechanical thromboprophylaxis

2. Mechanical thromboprophylaxis until the patient is ambulating

3. Mechanical thromboprophylaxis for the duration of hospital stay

4. Mechanical thromboprophylaxis for the duration of hospital stay and $\leq 2$ wk after discharge

5. Mechanical thromboprophylaxis for the duration of hospital stay and $>2$ wk after discharge 
Table 2 - VTE and major bleeding risk estimates of the presented patient profiles.

\begin{tabular}{|c|c|c|c|c|}
\hline Scenario & Operation & Patient risk strata ${ }^{a}$ & Risk of VTE $(\%)^{b}$ & Risk of major bleeding (\%) ${ }^{\mathrm{c}}$ \\
\hline 1 & Open radical cystectomy & High risk & 11.6 & 0.3 \\
\hline 2 & Open radical prostatectomy without lymphadenectomy & Low risk & 1.0 & 0.1 \\
\hline 2 & Robotic radical prostatectomy without lymphadenectomy & Low risk & 0.2 & 0.4 \\
\hline 3 & Open radical nephrectomy & Medium risk & 2.2 & 0.1 \\
\hline \multicolumn{5}{|c|}{$\begin{array}{l}\text { VTE = venous thromboembolism. } \\
\text { a Patient risk stratification according to the European Association of Urology guideline on thromboproph } \\
\text { b VTE defined as symptomatic pulmonary embolus or deep vein thrombosis within } 30 \mathrm{~d} \text { of surgery [2]. } \\
\text { c Major bleeding defined as bleeding requiring reoperation within } 30 \mathrm{~d} \text { of surgery [2]. }\end{array}$} \\
\hline
\end{tabular}

we sent an e-mail before each round to inform urologists about the upcoming mail survey.

In Canada, the CUA board of directors, who are independent of study investigators, reviewed the proposal and approved the administration of survey at the CUA annual meeting without requirement for further ethics review. In Finland, the ethics committee of the Helsinki and Uusimaa Hospital District granted exemption from ethical review (R11110). In Japan, the ethics committee of the University of Fukui (Fukui, Japan; \#20150067) approved the protocol. The reporting of the study conforms to the STROBE statement [9].

\subsection{Statistical analysis}

For every patient profile, we calculated the proportion of use of (1) mechanical prophylaxis, (2) pharmacological prophylaxis, and (3) extended pharmacological prophylaxis of $\geq 2 \mathrm{wk}$ and tested for statistical significance using chi-square analysis. As the length of hospital stay varies substantially between countries (considerably longer in Japan than in Canada or Finland; Supplementary Tables 1 and 2), and therefore the implication of prophylaxis during hospital stay differs across jurisdictions (Supplementary material), definitions of extended prophylaxis corresponded to duration of hospital stay in the three countries (Table 1 and Supplementary Table 3). We used multivariable logistic regression adjusted for the included countries to determine whether the urologist profile (resident/consultant) was associated with any mechanical prophylaxis, any pharmacological prophylaxis, or extended prophylaxis. We report the odds ratio and $95 \%$ confidence interval for the multivariable logistic regression using a threshold $p$ value of $<0.05$. All analyses were performed in SPSS version 20 (IBM Corp., Armonk, NY, USA).

\section{Results}

The 570 urologists who participated in this survey (Table 3 ) represent an overall response rate of 54\% (Canada: 216 of 385, 57\%; Finland: 110 of 179, 61\%; and Japan: 244 of 487 , $50 \%$ ). Of the 570 eligible individuals who responded to the survey, 566 (99\%) responded to at least five out of six scenario questions. Most participants were men (89.6\% [511/570]); the median age of the participants was $43.0 \mathrm{yr}$ (interquartile range: $35-54)$ with a majority being consultants (82.5\% [470/570]).

Almost all respondents used mechanical prophylaxis for patients undergoing RC (Canada: 92\% [199/216], Finland: 96\% [106/110], Japan: 96\% [234/244], $p=0.13$; Table 4). More respondents reported using mechanical prophylaxis after an open RP in Japan (98\% [239/244]) than in Finland (88\% [158/179]) and Canada (79\% [171/216], $p<0.001)$. Similarly, after a robotic RP, the Japanese urologists reported a higher rate of mechanical prophylaxis (100\%) than the Canadian (81\% [175/216]) and Finnish urologists (75\% [83/110]; $p<$ 0.001 ). Japanese urologists also reported more use of mechanical prophylaxis after an RN (95\% [232/244]) compared with Canadian (84\% [181/216]) and Finnish (80\% [88/ 110], $p<0.001$ ).

Use of pharmacological prophylaxis of any duration after RC was more common in Canada and Finland (both 99\% [208/210, 109/110]) than in Japan (70\% [171/244], $p<0.001$; Table 4). Fewer Japanese respondents indicated that they would use pharmacological prophylaxis after an RP (open RP: $14 \%$ [34/244], robotic RP: 33\% [81/244]) compared with Canadian (open RP: 93\% [201/216], robotic RP: 90\% [194/ 216]) and Finnish (open RP: 88\% [97/110], robotic RP: 94\% [103/110]) respondents $(p<0.0001$ for both open and robotic RP). Similarly, more respondents reported using pharmacological prophylaxis after an RN in Finland (95\% [105/110]) and Canada (92\% [199/216]) than in Japan (20\% [49/244], $p<0.001)$.

Finnish and Canadian respondents were more likely to use extended pharmacological prophylaxis after RC (Finland: 98\% [108/110], Canada: 84\% [181/216]) than Japanese respondents (26\% [63/244], $p<0.001)$. It was uncommon for respondents in any country to use extended

Table 3 - Baseline characteristics of the respondents.

\begin{tabular}{lllll}
\hline & Canada & Finland & Japan & Total \\
\hline Response (\%) & $216 / 385(57)$ & $110 / 179(61)$ & $244 / 487(50)$ & $570(54)$ \\
Male (\%) & $194(89.8)$ & $91(82.7)$ & $226(92.6)$ & $511(89.6)$ \\
Female (\%) & $19(8.8)^{\mathrm{a}}$ & $19(17.3)$ & $18(7.4)$ & $56(9.8)$ \\
Age (yr), median (IQR) & $38.0(33-50)$ & $48.0(30-57.5)$ & $45.0(38-55)$ & $43.0(35-54)$ \\
Consultant (\%) & $152(70.4)$ & $97(88.2)$ & $221(90.6)$ & $470(82.5)$ \\
Resident (\%) & $62(28.7)$ & $13(11.8)$ & & $97(17.0)$ \\
\hline IQR $=$ interquartile range. & & & \\
a Three missing values regarding gender in the Canadian sample. & & & \\
\hline
\end{tabular}


Table 4 - Proportion (in percentages) of those with any duration of mechanical prophylaxis, any duration of pharmacological prophylaxis, and pharmacological prophylaxis of at least $2 \mathrm{wk}$ by procedure and country.

\begin{tabular}{|c|c|c|c|c|c|c|}
\hline & \multicolumn{3}{|c|}{ Open radical cystectomy } & \multicolumn{3}{|c|}{ Open radical prostatectomy } \\
\hline & \multirow[t]{2}{*}{ Mechanical, any \% (95\% CI) } & \multicolumn{2}{|c|}{ Pharmacological } & \multirow[t]{2}{*}{ Mechanical, any \% (95\% CI) } & \multicolumn{2}{|c|}{ Pharmacological } \\
\hline & & $\begin{array}{c}\text { Any } \\
\%(95 \% \mathrm{CI})\end{array}$ & $\begin{array}{l}\text { Extended } \\
\%(95 \% \mathrm{CI})\end{array}$ & & $\begin{array}{c}\text { Any } \\
\%(95 \% \mathrm{CI})\end{array}$ & $\begin{array}{l}\text { Extended } \\
\%(95 \% \mathrm{CI})\end{array}$ \\
\hline Canada & $92(87-95)$ & $99(96-100)$ & $84(78-88)$ & $79(70-86)$ & 93 (86-97) & $8(4-15)$ \\
\hline Finland & $96(89-98)$ & $99(94-100)$ & $98(93-100)$ & $88(67-97)$ & 88 (67-97) & $25(11-47)$ \\
\hline \multirow[t]{4}{*}{ Japan } & $96(93-98)$ & $70(61-73)$ & $26(21-32)$ & $98(92-99)$ & $14(9-22)$ & $3(1-8)$ \\
\hline & \multicolumn{3}{|c|}{ Robotic radical prostatectomy } & \multicolumn{3}{|c|}{ Open radical nephrectomy } \\
\hline & \multirow[t]{2}{*}{ Mechanical, any \% (95\% CI) } & \multicolumn{2}{|c|}{ Pharmacological } & \multirow[t]{2}{*}{ Mechanical, any \% (95\% CI) } & \multicolumn{2}{|c|}{ Pharmacological } \\
\hline & & $\begin{array}{c}\text { Any } \\
\%(95 \% \mathrm{CI})\end{array}$ & $\begin{array}{l}\text { Extended } \\
\%(95 \% \mathrm{CI})\end{array}$ & & $\begin{array}{c}\text { Any } \\
\%(95 \% \mathrm{CI})\end{array}$ & $\begin{array}{l}\text { Extended } \\
\%(95 \% \mathrm{CI})\end{array}$ \\
\hline Canada & $81(72-88)$ & $90(82-95)$ & $9(4-17)$ & $80(74-85)$ & $92(87-95)$ & $7(4-12)$ \\
\hline Finland & $75(62-84)$ & $94(84-98)$ & $11(5-21)$ & $84(76-90)$ & $95(89-98)$ & $43(34-53)$ \\
\hline Japan & $100(98-100)$ & $33(23-46)$ & $0(0-2)$ & 95 (91-97) & $20(15-25)$ & $1(0-3)$ \\
\hline
\end{tabular}

pharmacological prophylaxis after open RP (Japan: 3\% [7/ 244], Canada: $8 \%$ [17/216], Finland: 25\% [28/110]; $p<0001$ ) and robotic RP (Japan: 0\% [0/244], Canada: 9\% [19/216], Finland: $11 \%[12 / 110] ; p=0.02)$. Finnish respondents $(43 \%$ [47/110]) were more likely to use extended pharmacological prophylaxis after RN than Canadian (7\% [15/216]) or Japanese $(1 \%[2 / 244])$ respondents $(p<0.001)$.

Multivariable analysis, adjusted for the country, demonstrated no difference in resident versus consultant urologists' use of mechanical, pharmacological, or extended pharmacological prophylaxis (Supplementary Table 4).

\section{Discussion}

This is the first study in urology to examine international variability in the use of thromboprophylaxis between countries in different continents. This large-scale multinational survey identified large variation in the use of pharmacological VTE prophylaxis during hospital stay and in the use of extended prophylaxis after discharge within and between countries. Canadian and Finnish urologists reported similar frequent use of pharmacological prophylaxis, but Japanese respondents reported far less use. Reported use of mechanical prophylaxis for urological procedures had much less variation, both within and between countries. Finally, we found no differences between resident and consultant urologists in the use of mechanical, pharmacological, or extended pharmacological prophylaxis.

\subsection{Strengths and limitations}

The strengths of the current study include a population representative of the target populations of Canadian, Finnish, and Japanese urologists, in age and gender distribution [10-12]. Our study's strengths also include a satisfactory participation rate and very high completeness of questionnaire responses. We assessed the use of thromboprophylaxis by clinical case scenarios relevant to current clinical practice.

Our study has limitations. First, as this survey was intended to capture an overview of practice pattern, we are not able to draw conclusion about the causes of the practice variation. We did not ask respondents about their perception of the incidence of VTE-perception of a lower risk in Japan could be responsible for a lower use of pharmacological, but not mechanical, prophylaxis. Whether such differences in incidence actually exist has not been established [2]. Second, how urologists actually practice in a hospital setting might differ from their responses to the scenarios presented in our study. In addition, as thromboprophylaxis is often prescribed by more junior doctors rather than by the operating surgeon, we did not collect data on the specialization of respondents. It is uncertain whether this approach biased estimates. Third, we have used different sampling strategies for Canada from those used for Finland and Japan. This pragmatic decision was based on our goal of including a representative sample in each country. Fourth, many of the respondents do not perform some of the procedures presented. They may, however, still participate in the prescription of thromboprophylaxis. Fifth, we assessed only a limited range of surgeries and scenarios. Fifth, the length of hospital stay was longer in Japan, which may influence the use of extended prophylaxis. Finally, generalization to jurisdictions beyond the three that we included in this survey remains uncertain.

\section{2. $\quad$ Comparison with other studies}

There are few earlier surveys examining the use of perioperative VTE prophylaxis. A British survey [5], on a mixed population of respondents including 29 consultant 
urologists, 30 residents, and 35 urology clinical nurse specialists practicing in $64 \mathrm{UK}$ pelvic cancer centers, found that all units used low-molecular-weight heparin (LMWH) prophylaxis routinely for the inpatient period after RC and 98\% used perioperative prophylaxis after RP. Routine use of LMWH for all patients after discharge following RC was reported in $67 \%$ and after RP in $61 \%$ of units (investigators did not differentiate between approaches, such as open or robotic) [5].

A US database study of 94709 men who underwent RP ( $72 \%$ open and $28 \%$ robotic) found that $52 \%$ of men after open $\mathrm{RP}$ received mechanical only, $7 \%$ pharmacological only, and $11 \%$ both mechanical and pharmacological prophylaxis, and 30\% received no prophylaxis [6]. Discharge prophylaxis was not included in the scope of this study. Another US study [4] surveyed the members of the American Urological Association in 2011. Only $11 \%$ of those invited responded (1210 respondents), of whom approximately $70 \%$ for RC and $<60 \%$ for RP reported the use of any thromboprophylaxis "frequently" or "always" [4]. The authors did not address postdischarge prophylaxis. Although small sample size [5], retrospective database design [6], and low response rate [4] limit the strength of inference from these studies, consistent with our finding, there remains little doubt of considerable variation in practice of thromboprophylaxis in urological procedures both within and between countries.

\subsection{Implications of findings}

The practice variation that we identified is likely due to several factors. First, the quality of evidence supporting decisions regarding VTE prophylaxis is not of high quality and thus open to variable interpretation [2,13]. Second, the low-quality evidence may also explain the divergent recommendations from several major VTE guidelines in different countries [8]. For instance, prominent VTE guidelines from the American College of Chest Physicians and National Institute for Health and Care Excellence do not consider specific urological surgeries separately but would consider all scenarios in the survey as "abdominopelvic" [14,15]. Third, urologists in some regions may also prefer to defer decisions regarding the use of thromboprophylaxis to colleagues from other disciplines with a different weighing of trade-off between bleeding and VTE from a nonsurgeon point of view. Possibly, VTE risk may also vary by population, given differences in body mass index or age at surgery that may predominate in different regions [14], with choices of prophylaxis appropriately reflecting local risk.

We performed the survey prior to the publication of the systematic reviews addressing estimates of absolute risks of symptomatic VTE and major bleeding in urological surgery $[2,13]$ and an EAU guideline addressing thromboprophylaxis [3], the first procedure and patient risk factor-specific guideline in urology. Therefore, respondents could not have been influenced by these guideline recommendations, and this survey can be used as a benchmark for tracking changes in practice pattern with further dissemination of urologyspecific VTE guidelines. Healthcare professionals across jurisdictions may consider, in their clinical decision making, this evidence-based, procedure-specific guideline [3], which has also been published as a freely available infographic [15]. Doing so would rationalize the practice and may decrease this substantial variation.

In patients at high risk of VTE, for some major surgeries, there is a clear net benefit to extended pharmacological thromboprophylaxis $[2,3,16]$. Our results suggest that for high-risk VTE and low bleeding risk (scenario 1: cystectomy for high-risk patients, Tables 1 and 2), practice in Canada and Finland follows the EAU guideline more closely [3].

In scenario 2 (RP without lymphadenectomy for lowrisk patients; Tables 1 and 2), the EAU guideline suggests the use of extended pharmacological prophylaxis in the open approach but recommends against the use of prophylaxis in robotic prostatectomy without lymphadenectomy [3]. In our survey, participants from Canada and Finland typically reported the use of pharmacological prophylaxis in hospital but not after discharge irrespective of the approach (open vs robotic), whereas one in seven of Japanese urologists after an open approach and one in three after a robotic approach reported in-hospital use of pharmacological prophylaxis.

In scenario 3 (open $\mathrm{RN}$ for medium-risk patients; Tables 1 and 2), respondents' practice in this situation proved to be less aligned with EAU guideline recommendations [3]: Canadian and Finnish respondents tended to report the use of in-hospital prophylaxis but not discharge prophylaxis, while Japanese respondents typically did not report any use of pharmacological prophylaxis.

\subsection{Unanswered questions and future research}

Variation in practice suggests limited adherence to clinical guidelines, which should represent the best practice for most patients. Therefore, efforts to increase familiarity with EAU guidelines [3] - rigorously evidence-based and the first procedure-specific guideline-may improve patient care. Perioperative VTE guidelines depend on a clear understanding of patient-important trade-offs and the quality of supporting evidence. Therefore, future efforts to improve the body of evidence, especially with the conduct of highquality trials and observational studies, will result in improved clinical guidance and patient care $[3,17,18]$. In addition, future surveys are needed to monitor changes in practice regarding perioperative thromboprophylaxis resulting from publication of new guidance.

\section{Conclusions}

We performed a large-scale multinational survey and identified a large variation in the use of pharmacological VTE prophylaxis within and between countries. This variation existed in the use of both pharmacological prophylaxis during hospital stay and extended prophylaxis after discharge. The variation in the reported use of mechanical prophylaxis for urological procedures was much less, with uniformly high use over $75 \%$ across all scenarios. Knowledge translation of evidence-based guidelines may reduce 
problematic variation in practice globally and thus may help optimize future patient care.

\author{
Author contributions: Kari A.O. Tikkinen had full access to all the data in \\ the study and takes responsibility for the integrity of the data and the \\ accuracy of the data analysis. \\ Study concept and design: Violette, Agarwal, Cartwright, Tailly, Novara, \\ Craigie, Guyatt, Tikkinen. \\ Acquisition of data: Violette, Aoki, Agarwal, Arai, Breau, Tikkinen. \\ Analysis and interpretation of data: All authors. \\ Drafting of the manuscript: Violette, Vernooij, Guyatt, Tikkinen. \\ Critical revision of the manuscript for important intellectual content: All \\ authors. \\ Statistical analysis: Vernooij. \\ Obtaining funding: Tikkinen. \\ Administrative, technical, or material support: None. \\ Supervision: Tikkinen. \\ Other: None.
}

Financial disclosures: Kari Tikkinen certifies that all conflicts of interest, including specific financial interests and relationships and affiliations relevant to the subject matter or materials discussed in the manuscript (eg, employment/affiliation, grants or funding, consultancies, honoraria, stock ownership or options, expert testimony, royalties, or patents filed, received, or pending), are the following: Violette has received an honorarium from Janssen, Sanofi, and Astellas prior to January 2017. Tailly has been a consultant for Cook Medical. Novara has been an advisory board member and speaker for Astellas, GlaxoSmithKline, Lilly, Menarini, Nycomed, Pfizer, Pierre Fabre, and Recordati. Vernooij, Aoki, Agarwal, Cartwright, Arai, Baldeh, Craigie, Breau, Guyatt, and Tikkinen have no financial conflicts of interest. Other potential conflicts of interests: Violette, Novara, Cartwright, and Guyatt were panel members and Tikkinen was the chairman of the European Association of Urology (EAU) Guideline on Thromboprophylaxis in Urological Surgery.

Funding/Support and role of the sponsor: The ISTHMUS project was conducted by the Clinical Urology and Epidemiology (CLUE) Working Group supported by the Academy of Finland (276046 and 309387), Competitive Research Funding of the Helsinki and Uusimaa Hospital District (TYH2016135, TYH2017114, TYH2018120, and TYH2019321), and Sigrid Jusélius Foundation. Philippe D. Violette was also supported by the Canadian Urological Association Scholarship Foundation and Rufus Cartwright by a Research Training Fellowship from the UK Medical Research Council. The sponsors had no role in the analysis and interpretation of the data or in the manuscript preparation, review, or approval.

Acknowledgements: We would like to thank the Tiffany Pizioli and the Canadian Urological Association administrative team, secretary of the Finnish Urological Association Sami Raatikainen, and research nurses Katja Kiianlinna and Merja Rignell, as well as the Japanese Urological Association Board of Directors, Syunsaku Tsukui, and Eiko Kawai for facilitating the surveys. We would also like to thank Joseph Chin, Jon Izawa, Sanna Myrskysalo, Nicholas Power, Jukka Sairanen, and Henrikki Santti for advice and support on estimating the timing of ambulation, duration of hospital stay, and representativeness of the study population.

\section{Appendix A. Supplementary data}

Supplementary material related to this article can be found, in the online version, at doi:https://doi.org/10. 1016/j.euf.2020.05.015.

\section{References}

[1] Anderson DR, Morgano GP, Bennett C, et al. American Society of Hematology 2019 guidelines for management of venous thromboembolism: prevention of venous thromboembolism in surgical hospitalized patients. Blood Adv 2019;3:3898-944.

[2] Tikkinen KAO, Craigie S, Agarwal A, et al. Procedure-specific risks of thrombosis and bleeding in urological cancer surgery: systematic review and meta-analysis. Eur Urol 2018;73:242-51.

[3] Tikkinen KAO, Cartwright R, Gould MK, et al. EAU guidelines on thromboprophylaxis in urological surgery. http://uroweb.org/ guideline/thromboprophylaxis/.

[4] Sterious S, Simhan J, Uzzo RG, et al. Familiarity and self-reported compliance with American Urological Association best practice recommendations for use of thromboembolic prophylaxis among American Urological Association members. J Urol 2013;190:992-8.

[5] Pridgeon S, Allchorne P, Turner B, Peters J, Green J. Venous thromboembolism (VTE) prophylaxis and urological pelvic cancer surgery: a UK national audit. BJU Int 2015;115:223-9.

[6] Weinberg A, Wright J, Deibert C, et al. Nationwide practice patterns for the use of venous thromboembolism prophylaxis among men undergoing radical prostatectomy. World J Urol 2014;32:1313-21.

[7] Tikkinen KA, Agarwal A, Craigie S, et al. Systematic reviews of observational studies of risk of thrombosis and bleeding in urological surgery (ROTBUS): introduction and methodology. Syst Rev 2014;3:150.

[8] Violette PD, Cartwright R, Briel M, Tikkinen KA, Guyatt GH. Guideline of guidelines: thromboprophylaxis for urological surgery. BJU Int 2016;118:351-8.

[9] von Elm E, Altman DG, Egger M, et al. The Strengthening the Reporting of Observational Studies in Epidemiology (STROBE) statement: guidelines for reporting observational studies. Lancet 2007;370:573-7.

[10] Canadian Urological Association (CUA) Membership Directory for 2017. https://www.cua.org/en/membership-directory.

[11] National Supervisory Authority for Welfare and Health (Valvira), Finland. Registers of social welfare and healthcare professionals. https://julkiterhikki.valvira.fi/\#.

[12] Ministry of Health, Labour and Welfare, Japan. Survey of physicians, dentists and pharmacists. https://www.mhlw.go.jp/toukei/saikin/ hw/ishi/16/index.html.

[13] Tikkinen KAO, Craigie S, Agarwal A, et al. Procedure-specific risks of thrombosis and bleeding in urological non-cancer surgery: systematic review and meta-analysis. Eur Urol 2018;73:236-41.

[14] Gould M, Garcia D, Wren S, et al. Prevention of VTE in nonorthopedic surgical patients: antithrombotic therapy and prevention of thrombosis, 9th ed: American College of Chest Physicians evidencebased clinical practice guidelines. Chest 2012;141, e227S-77S.

[15] National Institute for Health and Care Excellence. Venous thromboembolism in over 16s: reducing the risk of hospital-acquired deep vein thrombosis or pulmonary embolism. NICE guideline [NG89]. https://www.nice.org.uk/guidance/ng89.

[16] Heit JA, Spencer FA, White RH. The epidemiology of venous thromboembolism. J Thromb Thrombolysis 2016;41:3-14.

[17] Clinical Urology and Epidemiology Working Group. Infographic of the European Association of Urology (EAU) guideline on thromboprophylaxis in urological surgery. http://clueworkinggroup.com/ 2017/12/01/thromboprophylaxis-infographic/.

[18] Tikkinen Kari A O, Guyatt Gordon G H. Baseline risks of venous thromboembolism and major bleeding are crucial in thromboprophylaxis decision-making. Eur Urol 2020. http://dx.doi.org/10.1016/ j.eururo.2020.05.032. 